\title{
Power Minimization in a Backlit TFT-LCD Display by Concurrent Brightness and Contrast Scaling
}

\author{
Wei-Chung Cheng, Yu Hou, Massoud Pedram \\ Department of EE-Systems, University of Southern California \\ Los Angeles, CA 90089, USA \\ \{wccheng, yhou, massoud\}@sahand.usc.edu
}

\begin{abstract}
This paper presents a Concurrent Brightness and Contrast Scaling (CBCS) technique for a cold cathode fluorescent lamp (CCFL) backlit TFT-LCD display. The proposed technique aims at conserving power by reducing the backlight illumination while retaining the image fidelity through preservation of the image contrast. First, we explain how CCFL works and show how to model the non-linearity between its backlight illumination and power consumption. Next, we propose the contrast distortion metric to quantify the image quality loss after backlight scaling. Finally, we formulate and optimally solve the CBCS optimization problem with the objective of minimizing the fidelity and power metrics. Experimental results show that an average of $3.7 X$ power saving can be achieved with only $10 \%$ of contrast distortion.
\end{abstract}

\section{Introduction}

Previous studies on battery-powered electronics point out that the cold cathode fluorescent lamp (CCFL) backlight of an LCD display dominates the energy consumption of the whole system [1]. In the SmartBadge system, for instance, the display consumes $28.6 \%, 28.6 \%$, and $50 \%$ of the total power in the active, idle, and standby modes, respectively [1]. To reduce the power consumed by the backlight, researchers [2][3] have proposed the concept of Backlight Scaling. The backlight scaling technique dynamically dims the backlight to conserve its power consumption while increasing the transmissivity of the LCD panel to compensate for the image fidelity loss due to reduced backlight. Image fidelity is defined as the resemblance between the original and backlight-scaled image. If the backlight-scaled image is identical to the original image in terms of the brightness of each pixel (these approaches are called brightness-invariant backlight scaling), then there is no fidelity loss after backlight scaling. However, when a more aggressive backlight scaling policy is used to gain greater power savings, the brightness invariance is no longer attainable and the induced distortion degrades the image fidelity. Image fidelity can be measured by the brightness variance after backlight scaling. However, the

This research was supported in part by DARPA PAC/C program under contract DAAB07-02-C-P302 and by NSF under grant no. 9988441. brightness metric is too strict for efficacious backlight scaling policies. Since dimming the backlight directly limits the dynamic range of the image brightness, a brightness-invariant backlight scaling policy is usually too conservative to deliver great energy savings. In this paper, we propose using the image contrast as a metric to measure the image fidelity after backlight scaling.

\subsection{Terminology}

The following photometric quantities are illustrated around a backlit TFT-LCD display in Figure 1. Luminous flux (lumen) is the emission rate of light energy corrected for the standardized spectral response of human vision. Luminous intensity (candela) is defined as one lumen of luminous flux per steradiam ( $s r$-- unit of solid angle). Luminous intensity can be used to characterize the optical power emitted from a spot light source, such as a light bulb. Illuminance (lux) is defined as one lumen of luminous flux per area $\left(\mathrm{cd} / \mathrm{m}^{2}\right)$. Illuminance can be used to characterize the luminous power emitted from a surface. Most light meters (e.g., for photographic purpose) measure the illuminance quantity. The luminous flux may not travel in parallel after passing the surface, so that the light intensity decreases as the travel distance increases. Luminance (nit) is defined as lumen per area per steradiam $\left(\mathrm{lm} / \mathrm{m}^{2} / \mathrm{sr}\right)$. Luminance is used to rate the maximum brightness of CRT or LCD monitors [4].

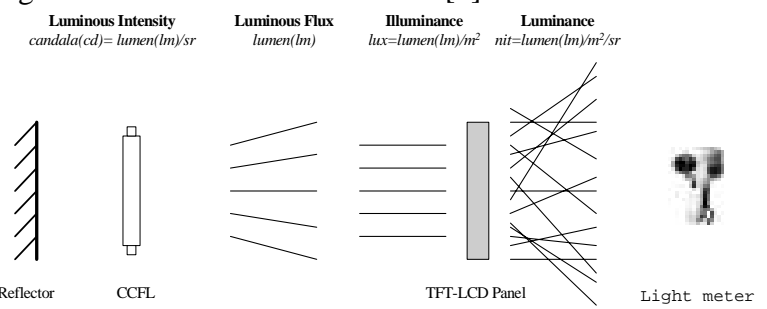

Figure 1: Illustration of CCFL backlight and photometric terms.

In this paper, we use backlight factor to express the percentage of the backlight illumination, and transmissivity to express the translucence of the TFT-LCD. The backlight factor and TFT-LCD transmissivity determine the perceived luminance from the TFT-LCD display.

\subsection{Backlit TFT-LCD display}

The major components of a backlit (or transmissive) TFTLCD display subsystem include the video controller, frame buffer, video interface, TFT-LCD panel, and backlight. The 
frame buffer is a portion of memory used by software applications to deliver video data to the video controller. The video data from the application is stored in the frame buffer by the CPU. The video controller fetches the video data and generates appropriate analog (VGA) or digital (DVI) video signals to the video interface. The video interface carries the video signals between the video controller and the TFT-LCD display. The TFT-LCD display receives the video data and generates proper shade - transmissivity - for each pixel according to its pixel value. All of the pixels on the transmissive LCD panel are illuminated by the backlight from behind. To the observer, a displayed pixel looks bright if its transmissivity is high (i.e., in the 'on' state), meaning it passes the backlight. On the other hand, a displayed pixel looks dark if its transmissivity is low (i.e., in the 'off' state), meaning it blocks the backlight. If the transmissivity can be adjusted to more than two different levels between the 'on' and 'off' states, then the pixels can be displayed in grayscale. If the shade can be colored as red, green, or blue by using different color filters, then pixels can be displayed in color by mixing three sub-pixels in different colors at different grayscales. In other words, the perceived brightness of a pixel is determined by its transmissivity and the backlight illumination.

Most of current TFT-LCD displays use CCFL backlighting thanks to its unrivaled luminance density - emitting the most light within the minimum form factor. The CCFL can be designed to generate arbitrary color, which is critical to reproducing pure white in the backlighting applications. The technology of manufacturing CCFL is mature so that its cost has been minimized. The power consumption of the CCFL backlight, however, is considerably high compared with that of the TFTLCD panel.

The observed luminance of a transmissive object $L$ is the product of the luminance of the light source $b$ and the transmissivity of the object $t$ [5]. For a pixel on a backlit TFTLCD display, its transmissivity is a function of its pixel value $x$. Thus, its observed luminance $L$ is

$$
L=t(x) \cdot b
$$

The ambient light is not considered here because it has little effect for a transmissive TFT-LCD when compared with a reflective or transflective one. Figure 2 depicts the relation in Equation (1) assuming that the transmissivity is a linear function of the pixel value.

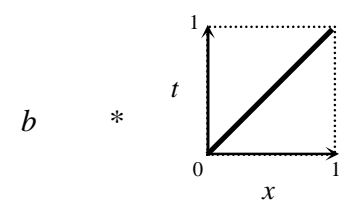

TFT-LCD Transimissivity Function

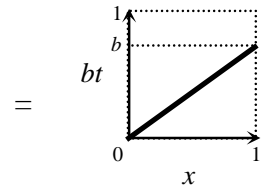

Luminance Function
CCFL

Backligh Factor

Figure 2: The luminance function of normalized pixel value (right) is the product of the backlight factor $b$ and the TFT-LCD transmissivity function (center).

In a non-backlight-scaled TFT-LCD display, the backlight $b$ is always fixed at full power. The backlight scaling techniques in [2][3] reduce $b$ while increasing the pixel value from $x$ to $x^{\prime}$ by

$$
\begin{aligned}
& x^{\prime}=x+b \\
& x^{\prime}=x / b,
\end{aligned}
$$

by maintaining the same $L$. These approaches have the following drawbacks:

- Equation (2) cannot preserve brightness invariance according to Equation (1).

- The contrast distortion among the unsaturated pixels is not considered.

- The software-based approach has high energy/performance overhead.

- The CCFL illumination is incorrectly modeled as a linear function of power.

In this paper, we propose solutions to surmount the abovementioned drawbacks. In Section 2 we characterize the CCFL illumination and power consumption. In Section 3 we propose adjusting the transmissivity function $t$ rather than the pixel value $x$. The optimal CBCS problem is introduced in Section 4. Section 5 presents the experimental results followed by conclusions in Section 6.

\section{CCFL Illumination and Power Modeling}

A CCFL backlight unit consists of the fluorescent lamp, the driving DC-AC inverter, and the light reflector. A CCFL is a sealed glass tube with electrodes on both ends. The tube is filled with an inert gas (argon) and mercury. The inner glass surface of the tube is coated with phosphor, which emits visible light when excited by photons. The wavelength or color of the visible light depends on the type of the gas and phosphor. In the LCD backlighting application, a proper mix of red, green, and blue phosphors produces the desired three-band white light. Otherwise, the displayed image will be color-shifted.

The CCFL converts electrical energy into visible light, which is called the gas discharge phenomenon. When a high voltage is applied to the electrodes turning on the lamp, electrical arcs are generated that ionize the gas and allow the electrical current to flow. The collision among the moving ions injects energy to the mercury atoms. The electrons of the mercury atoms receive energy and jump to a higher energy level followed by emitting ultraviolet photons when falling back to their original energy level. The ionized gas conducts the electrical current. The impedance of the gas conductor, unlike that of the metal conductor having a linear behavior, decreases as the current increases. Therefore, the CCFL has to be driven by an alternative current (AC) to avoid a potential explosion.

A $D C$-AC inverter is usually used to drive a CCFL in batterypowered applications. A DC-AC inverter is basically a switching oscillator circuit that supplies high-voltage AC current from a low-voltage battery. The nominal AC frequency of modern CCFL is in the range of $50-100 \mathrm{kHz}$ to avoid flickering. The nominal operate voltage has to be higher than $500 \mathrm{~V}_{\mathrm{RMS}}$ to keep inert gas ionized.

To conserve energy in battery-powered applications, dimming control is a desired feature for DC-AC inverters. Different methods of dimming CCFL have been used, including linear current, pulse-width-modulation, and current chopping [6]. In a DC-AC inverter with dimming control, an analog or digital input signal is exposed for adjusting the CCFL illumination. Most well designed DC-AC inverters have high electrical efficiency (>80\%) and linear response of output electrical power to input power. Most fluorescent lamps, however, have low optical efficiency $(<20 \%)$ and non-linear response of output optical power versus input power [7]. 


\subsection{CCFL Characteristics}

The CCFL illumination is a complex function of the driving current, ambient temperature, warm-up time, lamp age, driving waveform, lamp dimensions, and reflector design [7]. For CBCS, only the driving current is controllable. Therefore, we model the CCFL illumination as a function of the driving current only and ignore the other parameters.

The typical relationship between the CCFL illumination and the driving power is shown in Figure 3a. The CCFL illumination increases monotonically as the driving power increases before reaching $80 \%$ of the full driving power. Beyond $80 \%$, the CCFL illumination starts to saturate. This saturation phenomenon is because the enclosed ionized gas has been fully discharged and cannot release more photons. Additionally, the increased temperature and pressure inside the tube inhibit further discharge [7][8]. This observation suggests that the decreased optical efficiency of CCFL in the saturated region is not favored by power-aware applications.

\subsection{CCFL Illumination/Power Characterization}

We use a stepwise function of illumination to characterize the power consumption of CCFL as a function of illumination:

$$
P_{\text {backlight }}(b)=\left\{\begin{array}{ll}
b \cdot P_{\text {Lin }}+C_{\text {Lin }}, & 0 \leq b \leq B_{S} \\
b \cdot P_{\text {Sat }}+C_{\text {Sat }}, & B_{S} \leq b \leq 1
\end{array}\right. \text { (Watt). }
$$

The backlight factor $b \in[0,1]$ represents the normalized backlight illumination, which is dynamically controllable by the CBCS policy.

The analog or digital dimming control input of the DC-AC inverter is not always linearly proportional to the output backlight illumination. Careful calibration is needed to derive the correct mapping between the backlight factor $b$ and dimming control input $q(b)$. A precision luminance meter such as that in [9] provides accurate absolute illuminance readings. These expensive meters, however, are commonly unavailable to electronic laboratories. We find that the absolute illuminance readings are not required to calibrate the CCFL in the backlight scaling applications. An accurate photographic light meter can serve the purpose so far as it is capable of sensing minor luminance variance. We use the light meter as a weight scale and adjust the backlight and TFT-LCD simultaneously while maintaining the same illuminance. We start with measuring the illuminance for the maximum CCFL backlight $b=1$ when applying dimming control $q(b=1)$ and minimum LCD transmissivity $x=0 \in[0,255]$. The transmissivity $x$ is obtained by displaying a pure gray image, in which $\operatorname{Red}=$ Green $=$ Blue $=x$ for every pixel. The transmissivity $x$ is increased until the light meter can sense a variation and report a different reading. Then reduce the backlight factor $b$ by reducing the dimming control $q$ until the meter reports the previous reading. Since the change of the TFTLCD grayscale (transmissivity) is known, the change of the backlight is asserted to be the same. Record $q$ as the dimming control value for the backlight factor $b=(255-x) / 256$. At the same time, the power consumption of the backlight $P_{\text {backlight }}$ is also measured and recorded. Repeat the above procedure for $x=0 . .255$. After interpolation, we can obtain $q(b)$ and $P_{\text {backlight }}(b)$. The results for a color backlit TFT-LCD [10] are shown in Figure 3a. Plugging into Equation 4, the following parameters are obtained: $P_{\text {Lin }}=0.4991, P_{\text {Sat }}=0.1489, C_{\text {Lin }}=0.1113, C_{\text {Sat }}=0.6119, B_{s}=0.8666$

This power model will be incorporated in Section 4 to solve the optimal CBCS problem.
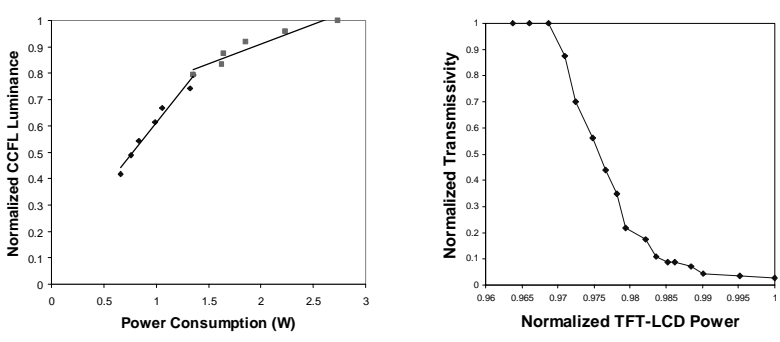

Figure 3: (a) Luminance/Power characterization of CCFL (b) Transmissivity/Power characterization of TFT-LCD panel.

\section{TFT-LCD Grayscale Control and Power Modeling}

In a TFT-LCD display, each sub-pixel has an individual liquid crystal cell, a thin-film-transistor (TFT) and a capacitor. The electrical field of the capacitor controls the orientation of the liquid crystals within the cell, which indeed determines the transmissivity. The capacitor is charged and discharged by its own TFT. The gate electrode of the TFT controls the timing of charging/discharging when the pixel is scanned for refreshing its content. The source electrode of the TFT controls the amount of charge that determines the transmissivity of the liquid crystal cell. The gate electrodes and source electrodes of all TFTs are driven by a set of gate drivers and source drivers, respectively. A single gate driver drives all gate electrodes of the pixels on the same row. The gate electrodes are enabled at the same time the row is scanned. A single source driver drives all source electrodes of the pixels on the same column. The source driver supplies the desired voltage level (called grayscale voltage) according to the pixel value. In other words, ideally, the transmissivity $t(v(x))$ is a linear function of the grayscale voltage $v(x)$, which is a linear function of the pixel value $x$. If there are 256 grayscales, then the source driver must be able to supply 256 different grayscale voltage levels. For the source driver to provide a wide range of grayscales, a number of reference voltages are required. The source driver mixes different reference voltages to obtain the desired grayscale voltages. Typically, these different reference voltages are fixed and designed as a voltage divider. For example in [10], an LCD reference driver [11] is used with a 10-way voltage divider. Assume that the transmissivity of the TFT-LCD is linear and the resistors of the voltage divider are identical. If $k$ identical resistors $r_{l} \ldots r_{k}$ are connected in series between $V_{k}$ and ground, then the output voltage from $r_{k}$ is

$$
V_{i}=\frac{i}{k} V_{k}
$$

\subsection{Programmable LCD Reference Driver}

Our approach to CBCS is to control the mapping of $v(x)$ in order to control the transmissivity function $t(x)$. We propose using a programmable LCD reference driver (PLRD) described as follows.

The PLRD is implemented by adding an extra logic to the original voltage divider expressed by Equation (6). The logic contains a number of p-channel and n-channel switches and multiplexers. The PLRD takes two input arguments $g l$ and $g u$, and then connects $r_{g u}, r_{g u+l} \ldots r_{k}$ to $V_{k}$ and $r_{0}, r_{l} \ldots r_{g l}$ to ground. In this way, the output voltage seen from $r_{k}$ becomes 


$$
V_{i, g l, g u}^{\prime}=\left\{\begin{array}{cc}
V_{k}, & g u \leq i \leq k \\
\frac{i-g l}{g u-g l} V_{k}, & g l \leq i<g u \\
0, & 0 \leq i \leq g l
\end{array} .\right.
$$

Clearly, the PLRD performs a linear transformation (limited by 0 and $V_{k}$ ) on the original reference voltages, and therefore, provides the CBCS policy a mechanism for adjusting the TFTLCD transmissivity function as shown in Figure 4a. The luminance function is shown in Figure $4 \mathrm{~b}$.

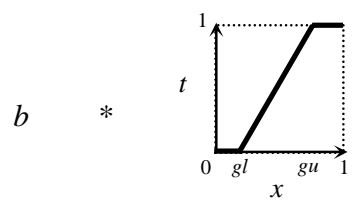

(a)

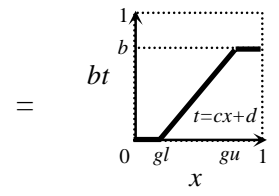

(b)
Figure 4: The transmissivity function (a) and luminance function (b) when using a programmable LCD reference driver.

The similar concept of PLRD has been implemented in TFTLCD controllers such as [12] to control contrast. The PLRD represents a class of linear transformations on the backlightscaled image. It covers both brightness scaling (adjusting $g u$ and $g l$ simultaneously) and contrast scaling (adjusting $g u-g l$ ). On the other hand, non-linear transformations are not desired in backlight scaling because they cannot preserve the uniformity of contrast.

\subsection{TFT-LCD Power Characterization}

The TFT power can be modeled by a quadratic function of pixel value $x \in[0,255][13]$ :

$$
P_{T F T}(x)=c_{0}+c_{1} x+c_{2} x^{2}(\text { Watt }) \text {. }
$$

We performed the current and power measurements on [10]. The measurement data are shown in Figure $3 \mathrm{~b}$. Plugging into Equation 8, the coefficients are found as:

$$
c_{0}=2.703 E-3, c_{1}=2.821 E-4, c_{2}=2.807 E-5 \text {. }
$$

The TFT-LCD power consumption decreases as the transmissivity increases. In other words, while maintaining the same luminance, the power consumption of the TFT-LCD decreases when dimming the backlight. In addition, the variation of TFT-LCD power consumption is very small. Therefore, we do not consider the TFT-LCD power consumption in the CBCS framework.

\section{Optimal CBCS Policy Problem}
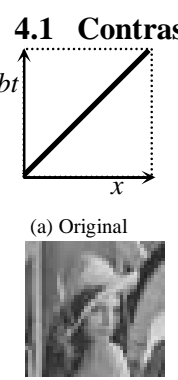
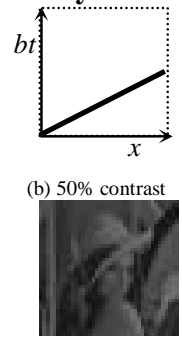

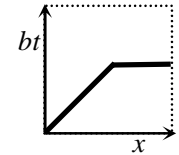

(c) $50 \%$ brightness

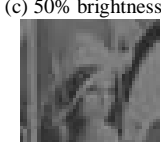

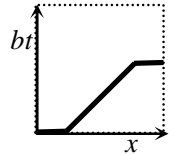

(d) $50 \% \mathrm{CBCS}$

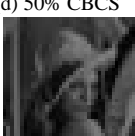

Figure 5: Luminance functions and visual effects of adjusting brightness (b), contrast (c), and both (d) when the backlight is dimmed to $50 \%$.
The term contrast describes the concept of the differences between the dark and bright pixels. Brightness and contrast are the two most important properties of any image. In the Human Visual System [5][14], which models the perception of human vision as a three-stage processing, the brightness and contrast are perceived in the first two stages. Virtually every single display permits the users to adjust the brightness and contrast settings. For backlit LCD displays, the brightness control changes the backlight illumination and the contrast control changes the LCD transmissivity function. Figure 5 shows how the brightness and contrast controls change the luminance function and their visual effects when the maximum brightness is limited to $50 \%$. In Figure $5 b$, when the backlight is reduced to $50 \%$, the image contrast is noticeably reduced. If we compensate for the contrast loss as shown in Figure 5c, then the darker $(<50 \%)$ pixels preserve their original brightness while the brighter $(>50 \%)$ pixels overshoot completely and there is no contrast present among these pixels. Figure 5d shows how the concurrent brightness and contrast scaling generates a better image by balancing the contrast loss and number of overshot pixels. The luminance function in Figure $5 \mathrm{~d}$ or Figure $4 \mathrm{~b}$ represents the following class of linear transformations that can be implemented by the PLRD expressed by Equation (7):

$$
b \cdot t(x)=\left\{\begin{array}{cc}
0, & 0 \leq x \leq g l \\
c x+d, & g l \leq x \leq g u, \\
b, & g u \leq x \leq 1
\end{array} \quad \text { where } \begin{array}{c}
g l=\frac{-d}{c} \\
g u=\frac{b-d}{c}
\end{array} .\right.
$$

Here $(g l, 0)$ and $(g u, b)$ are the points where $y=c x+d$ intersects $y=0$ and $y=b$, respectively. The luminance function consists of three regions: the undershot region $[0, g l]$, the linear region $[g l, g u]$, and the overshot region $[g u, 1]$. In other words, the $g l$ and $g u$ are the darkest and the brightest pixel values that can be displayed without contrast distortion (overshooting or undershooting). Notice that the slope of the linear region is very close to that of the original luminance function, which is unity. The image has very few pixels in the undershot and overshot regions. Its histogram is shown in Figure 6a.

The kernel of CBCS is to find the dissimilarity between the original and backlight-scaled image, which can be solely determined by examining the luminance function $b t(x)$. We define the contrast fidelity function as the derivative of $b t(x)$ :

$$
f_{c}(x)=\left\{\begin{array}{lc}
0, & 0 \leq x<g l \\
c, & g l \leq x \leq g u, \quad 0 \leq c \leq 1 \\
0, & g u<x \leq 1
\end{array} .\right.
$$

The $c$ is limited between 0 and 1 . If $c>1$, the contrast increases and deviates from that of the original image and the dynamic range $[g l, g u]$ shrinks. The overall contrast fidelity will decrease from this point, so we do not include $c>1$ in our solution space.

The contrast fidelity is defined without quantifying contrast itself, which has no universal definition [15] and cannot help solve the optimal CBCS policy problem. However, the definition of contrast fidelity does convey the concept of the classic definitions of contrast such as Weber's or Michelson's that express contrast as the ratio of the luminance difference to the maximum luminance [5][14][15]. If the normalized image histogram providing the probability distribution of the occurrence of pixel value $x$ in the image is given as

$$
p(x) \in[0,1], x=0 . .255 \text {, }
$$

then the global contrast fidelity of the backlight-scaled image is defined as 


$$
F_{C}=\sum_{g l}^{g u} f_{c}(x) \cdot p(x) .
$$

$F_{c}$ is a function of $p, g l$ and $g u$. Finding the optimal solution that minimizes the $F_{c}$ is called the optimal CBCS policy problem.

The global contrast fidelity captures the brightness distortion due to backlight scaling, also. When the backlight is dimmed, the dynamic range $[g l, g u]$ is shrunk accordingly, so that more pixels have contrast fidelity of zero.

\subsection{Contrast fidelity Optimization Problem}

To simplify the optimal CBCS policy problem, our approach is first to find the optimal linear transmissivity function for each given backlight factor, called the contrast fidelity optimization problem. Then we sweep the backlight factor domain to find the globally optimal solutions.

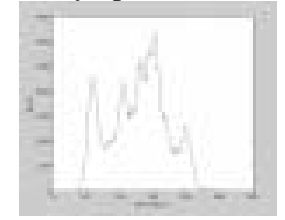

(a)

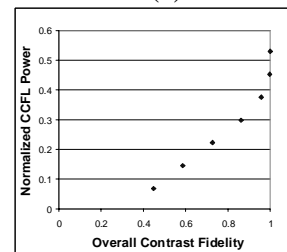

(d)

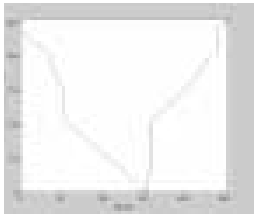

(b)

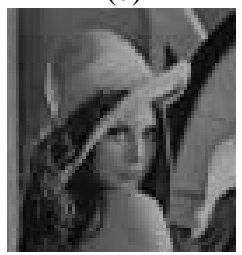

(e)

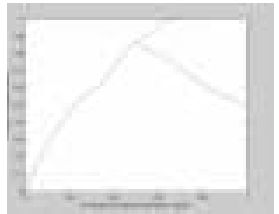

(c)

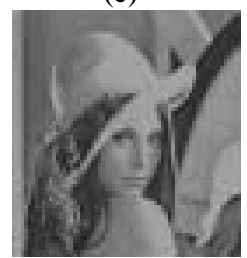

(f)
Figure 6: (a) Histogram of the example image (b) Optimal $g l$ (left) and $g l+d r$ (right) as functions of dynamic range $d r$ in the $y$ axis (c) Overall contrast fidelity $F_{c}$ as a function of dynamic range $d r$ for $b=1$ (upper) and $b=0.5$ (lower) (d) Optimal solutions $\left\langle F_{c}, P_{\text {backlight }}\right\rangle$ (e) CBCS policy (f) Brightness-invariant policy.

Our goal is to find the optimal $g l$ and $g u$ that maximize the overall contrast fidelity $F_{c}$. After that, the optimal coefficients $c$ and $d$ can be calculated from Equation (10). The optimal transmissivity function $t(x)$, which should be applied to the LCD as Figure $4 a$, can then be determined by

$$
t(x)=\left\{\begin{array}{cc}
0, & 0 \leq x<g l \\
\frac{c x+d}{b}, & g l \leq x \leq g u \\
1, & g u<x \leq 1
\end{array},\right.
$$

and the backlight should be dimmed to $b$ concurrently.

The optimal solution to the contrast fidelity optimization problem for an arbitrary histogram can be found by the following procedures.

Let $d r=g u-g l$ be the size of the required dynamic range $[g l, g u]$ and the backlight factor $b$ be the size of the available dynamic range $[0, b]$. For each $d r$, we can find the required dynamic range $[g l, g l+d r]$ that maximizes $\sum_{g l}^{g l+d r} p(x)$. The optimal $g l$ is found by scanning $g l=0 * 256 / k, 1 * 256 / k, \ldots(k-1) * 256 / k$, where $k$ represents the resolution of the PLRD in Equation (7). Based on the histogram shown in Figure 6a, Figure 6b shows the optimal $g l$ and $g l+d r$ in the $x$ axis as functions of $d r$ in the $y$ axis. The left and right curves are the optimal $g l$ and $g l+d r$, respectively, for different $d r$ values. This means when the backlight is dimmed to $d r$, using the available dynamic range $[0, d r]$ from the backlit
LCD to display the required dynamic range $[g l, g l+d r]$ by the image will generate a backlight-scaled image that minimizes the number of undershot or overshot pixels.

Now consider the contrast fidelity $c$ in Equation (10). If the available dynamic range is larger or equal to the required dynamic range $(d r \leq b)$, the optimal contrast fidelity $c=1$ can be obtained with $d \leq 0$ and the overall contrast fidelity $F_{c}$ is simply $\sum_{g l}^{g u} p(x)$. Otherwise, if $d r>b$, the highest possible contrast fidelity is $c=b / d r$ with $t=1$ and $d=0$. Thus, $F_{c}$ becomes

$$
\frac{b}{d r} \sum_{g l}^{g l+d r} p(x)
$$

Figure $6 \mathrm{c}$ shows $F_{c}$ as a function of $d r$ for $b=1$ (upper) and $b=0.5$ (lower). The $F_{c}$ increases as $d r$ increases from $d r=0$ to $d r=0.5$. For the $b=1$ curve, the example image needs no more than $70 \%$ of available dynamic range to represent the whole histogram with the best contrast fidelity $c=1$. For the $b=0.5$ curve, the $F_{c}$ decreases from $d r=0.5$ to $d r=1$ because in Equation (15) the $\sum_{g l}^{g l+d r} p(x)$ increases slower than $d r$. The optimal $F_{c}$ happens at $d r=0.5$ and the contrast fidelity $c=1$ in the region $[g l, g l+d r]$. Notice that $c=1$ is not always the optimal solution when $d r>b$. If the distribution in the histogram is not normal (e.g. has two peaks) the optimal $d r$ can be greater than $b$, such that $\sum_{g l}^{g l d r} p(x)$ can be increased. For each backlight factor $b$, the complexity of finding the optimal $F_{c}, g l$ and $g u$ is $O\left(k^{2}\right)$ with $k$ a small number $(<12)$.

\subsection{Fidelity-Power Optimization}

Given the solution to the contrast fidelity optimization problem for any backlight factor $b$, the optimal CBCS policy problem can be solved by sweeping the backlight factor range between $b_{\min }$ and $b_{\max }$, where $b_{\min }$ and $b_{\max }$ are user-specified minimum and maximum backlight factors, respectively. All of the optimal solutions are recorded along with their power consumptions. The inferior solutions, i.e., same fidelity but higher power or same power but lower fidelity, are discarded. The remaining solutions are stored for the CBCS policy to select the most suitable solution according to the user preferences. Figure $6 \mathrm{~d}$ shows the 7 optimal solutions for $b=0.8,0.7, \ldots 0.2$ from top to bottom. The $x$ and $y$ coordinates of each solution indicate the global contrast fidelity and backlight power, respectively. The two inferior solutions for $b=1.0$ and 0.9 are discarded because they have the same fidelity, $F_{c}=1$, as that of $b=0.8$. The results show that more than $50 \%$ power savings can be achieved by the CBCS policy while maintaining almost $100 \%$ of contrast fidelity at a backlight factor of $70 \%$. The visual effect is shown in Figure 6e, in comparison with Figure $6 \mathrm{f}$ generated from the brightness-invariant policy from Equation (3).

The procedures for the contrast fidelity optimized CBCS are summarized in Figure 7.

\section{Experimental Results}

We use a set of benchmark images from the USC SIPI Image Database (USID) 0. The USID is considered the de facto benchmark suite in the signal and image processing research field [5]. The results reported here are from 8 color images from volume 3 . All of them are 256 by 256 pixels. The color depth is 24 bits, i.e., 8 bits per color-channel in the range of 0 to 255 . 


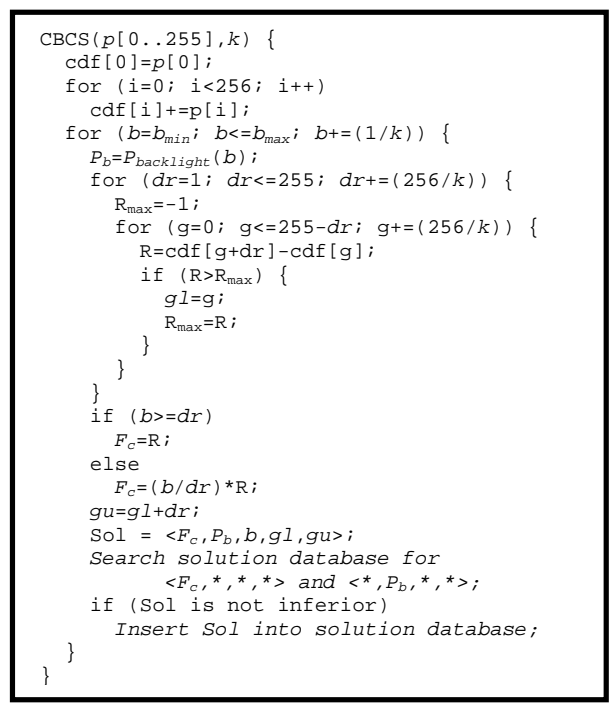

Figure 7: CBCS Optimization Flow.

Table 1: Optimal CBCS solutions to the USID benchmark

\begin{tabular}{|c|c|c|c|c|c|}
\hline $\begin{array}{l}\text { Image } \\
\#\end{array}$ & $\begin{array}{c}\text { Backlight } \\
\text { factor } \\
b\end{array}$ & $\begin{array}{c}\text { Contrast } \\
\text { fidelity } \\
c\end{array}$ & $\begin{array}{c}\text { Brightness } \\
\text { shift } \\
d\end{array}$ & $\begin{array}{c}\text { Overall } \\
\text { fidelity } \\
F_{c}\end{array}$ & $\begin{array}{l}\text { CCFL } \\
\text { Power } \\
(\mathrm{mW})\end{array}$ \\
\hline 4.1 .01 & 0.51 & 1 & 0.00 & 0.91 & 803.84 \\
\hline 4.1 .02 & 0.38 & 1 & 0.00 & 0.91 & 549.99 \\
\hline 4.1 .03 & 0.65 & 1 & 0.00 & 0.91 & 1077.21 \\
\hline 4.1 .04 & 0.75 & 1 & 0.00 & 0.91 & 1272.47 \\
\hline 4.1 .05 & 0.75 & 1 & 0.01 & 0.91 & 1272.47 \\
\hline 4.1 .06 & 0.84 & 1 & 0.04 & 0.90 & 1448.21 \\
\hline 4.1 .07 & 0.71 & 1 & 0.06 & 0.90 & 1194.36 \\
\hline 4.1 .08 & 0.72 & 1 & 0.04 & 0.92 & 1213.89 \\
\hline
\end{tabular}

Table 1 and 2 show the optimal CBCS policies for the benchmark images. We use 0.9 as the global contrast fidelity threshold to find the minimum backlight factor and its optimal linear transformation. The results show an average of $3.7 \mathrm{X}$ power savings within $10 \%$ of contrast distortion.

\section{Conclusions and Future Work}

We have presented the CBCS technique for a CCFL backlit TFT-LCD display. The proposed technique aims at conserving power by reducing the backlight illumination while retaining the image fidelity through preservation of the image contrast. We have explained how CCFL works and showed how to model the non-linearity between its backlight illumination and power consumption. We have proposed the contrast distortion metric to quantify the image quality loss after backlight scaling. We have formulated and optimally solved the CBCS optimization problem with the objective of minimizing the fidelity and power metrics. Experimental results show that an average of $3.7 \mathrm{X}$ power savings can be achieved with $10 \%$ of contrast distortion. The CBCS technique we propose in this paper is only for still images. Future studies, however, should consider applying it to video applications. Since the decision of the backlight factor is based on each frame individually, the backlight factor may change significantly across consecutive frames because the histogram varies significantly. The huge change in the backlight factor will introduce inter-frame brightness distortion to the observer. When the CBCS technique is to be applied to video applications such as an MPEG2 decoder, the change of the backlight factor should be limited such that the change is too subtle to be sensed by human eyes.

Table 2: Original images (upper) vs. backlight-scaled images (lower)

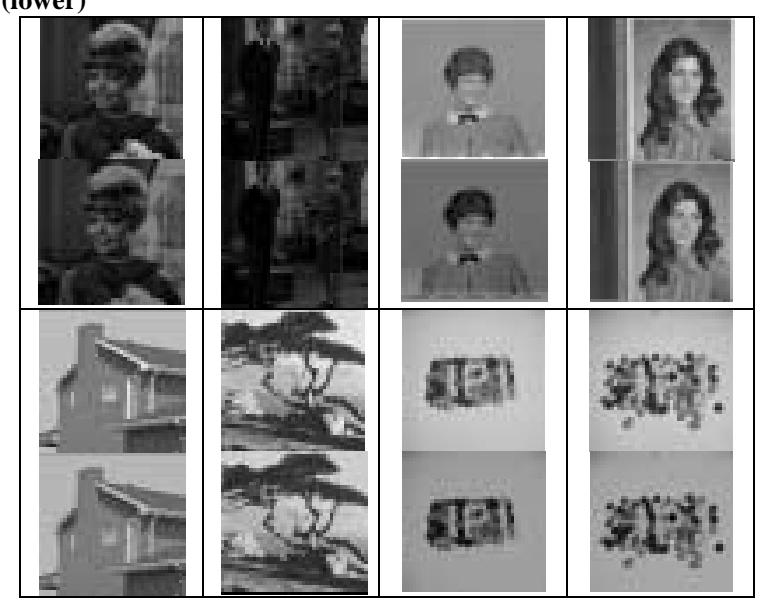

\section{References}

[1] T. Simunic et al, "Event-driven power management," IEEE Tran. Computer-Aided Design of Integrated Circuits and Systems, vol. 20, pp. 840-857, July 2001.

[2] I. Choi, H. Shim, and N. Chang, "Low-power color TFT LCD display for hand-held embedded systems," Proc. of Symp. on Low Power Electronics and Design, Aug. 2002, pp. 112-117.

[3] F. Gatti, A. Acquaviva, L. Benini, B Ricco, "Low power control techniques for TFT LCD displays," Proc. Intl. Conf. Compilers, Architecture, and Synthesis for Embedded Systems, October 2002, pp. 218-224.

[4] Robert L. Myers, Display Interfaces: Fundamentals and Standards, Chichester, England: Wiley, 2002.

[5] W. K. Pratt, Digital Image Processing, Wiley Interscience, 1991.

[6] Maxim, MAX1610 Digitally Controlled CCFL Backlight Power Supply.

[7] Jim Williams, "A fourth generation of LCD backlight technology," Linear Technology Application Note 65, Nov. 1995.

[8] Stanley Electric Co., Ltd., [CFL] cold cathode fluorescent lamps, 2003.

[9] Minolta, Minolta Precision Luminance Meter LS-100.

[10] LG Philips, LP064V1 Liquid Crystal Display.

[11] Analog Devices, AD8511 11-Channel, Muxed Input LCD Reference Drivers.

[12] Hitachi, HD66753 168x132-dot Graphics LCD Controller/Driver with Bit-operation Functions, 2003.

[13] H. Aoki, "Dynamic characterization of a-Si TFT-LCD pixels," HP Labs 1996 Technical Reports (HPL-96-19), February 21, 1996.

[14] S. Daly, "The visible differences predictor: an algorithm for the assessment of image fidelity," Digital images and human vision, pp. 179-206, Cambridge: MIT Press, 1993.

[15] E. Peli, "Contrast in complex images," J. Opt. Soc. Amer. A, vol. 10, no. 10, pp. 2032-2040, Oct. 1990.

[16] A. G. Weber, "The USC-SIPI Image Database Version 5," USCSIPI Report \#315, Oct. 1997. 